\title{
Correlation of increased MALAT1 expression with pathological features and prognosis in cancer patients: a meta-analysis
}

\author{
X.S. Shi ${ }^{1 *}$, J. Li ${ }^{2 *}$, R.H. Yang ${ }^{1}$, G.R. Zhao ${ }^{1}$, H.P. Zhou ${ }^{1}$, W.X. Zeng ${ }^{1}$ and M. Zhou ${ }^{1}$ \\ 'Department of Thoracic Surgery, Cancer Center of Guangzhou Medical University, \\ Guangzhou, Guangdong, China \\ ${ }^{2}$ State Key Laboratory of Respiratory Disease, \\ The First Affiliated Hospital of Guangzhou Medical University, \\ National Clinical Research Center for Respiratory Disease, China \\ *These authors contributed equally to this study. \\ Corresponding author: M. Zhou \\ E-mail: gzzmyp@126.com
}

Genet. Mol. Res. 14 (4): 18808-18819 (2015)

Received August 18, 2015

Accepted October 11, 2015

Published December 28, 2015

DOI http://dx.doi.org/10.4238/2015.December.28.30

ABSTRACT. Metastasis-associated lung adenocarcinoma transcript 1 (MALAT1) has been identified as a potential cancer biomarker, yet the mechanism by which it influences the development of cancer remains unknown. In this study, we aimed to correlate MALAT1 expression with pathological features and prognosis in cancer patients. Several databases were searched using combinations of keywords relating to MALAT1 and cancer. After selection of relevant cohort studies according to strict criteria, a meta-analysis was conducted. Twelve studies were analyzed, involving 958 cancer patients. Elevated MALAT1 expression was associated with poor prognosis and larger tumors [prognosis: hazard ratio $=3.11,95 \%$ confidence interval $(\mathrm{Cl})=1.98-4.23, \mathrm{P}=0.000$; tumor size: odds ratio $(\mathrm{OR})=0.40,95 \% \mathrm{Cl}=0.21-0.74, \mathrm{P}=0.003]$. However, no connection with histological grade, T-stage, lymph node (LN) metastasis, or distant metastasis was established (all $\mathrm{P}>0.05$ ). A correlation between increased 
expression and poor prognosis was observed in the large and small sample-size subgroups (all $\mathrm{P}<0.05$ ), as was a relationship with large tumor size $(\mathrm{OR}=0.30,95 \% \mathrm{Cl}=0.13-0.71, \mathrm{P}=0.006)$. Expression was correlated with T-stage and distant metastasis in the small sample-size subgroup (all $\mathrm{P}<0.05$ ), but no association was detected regarding histological grade, LN metastasis in either subgroup (all $\mathrm{P}>0.05$ ). Our findings demonstrate that elevated MALAT1 expression correlates with large tumor size, advanced tumor stage, and poor prognosis, and might therefore be utilized to evaluate clinical pathological features and prognostic out come for cancer patients.

Key words: MALAT1; Protein expression; Cancer; Pathological features; Prognosis; Meta-analysis

\section{INTRODUCTION}

Cancer, with its potential to develop in any organ or location in the human body, is considered a malignant disease owing to the fact that cancerous cells grow at an abnormal speed within tissues (Yamashita and Wang, 2013; Lapunzina et al., 2014). Due to the diversity of cancer types, signs and symptoms differ between tumors (Axon, 2006; Astin et al., 2011). Cancer has long been recognized as a serious threat to human health (Leong and $\mathrm{Ng}, 2014$ ). For example, lung cancers are the fifth most common cause of death worldwide of any disease, and gastric cancer has been categorized as the second most common cause of cancer-related death worldwide (Okugawa et al., 2014). Despite the application of significant systematic and sensitive medical care, the prognosis for patients with certain cancers remains poor, owing to unavoidable invasion and metastasis (Pang et al., 2015; Zhang et al., 2015). Therefore, medical research has focused on identifying factors that contribute to poor cancer prognosis (Yan et al., 2008; Gao et al., 2011). In recent years, long noncoding RNAs (IncRNAs) have been implicated in regulating the growth and apoptosis of human cells, and thus may contribute to carcinogenesis (Zhang et al., 2015). As a classic IncRNA, metastasis-associated lung adenocarcinoma transcript 1 (MALAT1) has been reported as a potential factor associated with unfavorable cancer prognosis (Dong et al., 2015; Pang et al., 2015).

MALAT1, also known as NEAT2, is approximately 8000 nucleotides in length and has been shown to be commonly expressed in humans (Tripathi et al., 2010; Zhang et al., 2015). It has previously been demonstrated that this IncRNA is capable of interacting with the demethylated form of chromo box homolog 4 ( $C B X 4$, also known as PC2), and that such interaction can regulate the re-localization of genes controlling growth. In addition, MALAT1 has been found to localize to subnuclear structures, areas of active or silent gene expression, and thus may be able to activate expression by interfering with the assembly of coactivator complexes (Gutschner et al., 2013). Recently, it has been proposed that MALAT1 expression may be involved in the progression and prognosis of various cancers, including pancreatic and colorectal malignancies (Zheng et al., 2014; Pang et al., 2015). Some reports also indicate that its differential expression may be linked with metastasis and recurrence of certain cancers (Liu et al., 2014; Zhang et al., 2015). These proposals may be based on the fact that MALAT1 is able to control the activity of the transcription factor E2F1, which plays a key role in cell cycle progression and tumorigenesis (Tripathi et al., 2013; Zheng et al., 2014). Moreover, MALAT1 has been observed to enhance the proliferation and migration of human cells by mediating the expression of pre-mRNA, demonstrating effects conducive to metastasis and transformation in tumor cells (Lai et al., 2012). When MALAT1 expression 
is knocked down, migration and invasion cell processes are repressed and G2/M cell-cycle arrest and cell apoptosis can subsequently be induced (Pang et al., 2015). Therefore, expression of this IncRNA can be seen to be correlated with the clinical features and prognosis of certain cancers. Nevertheless, recent studies related to its role in carcinogenesis have generated conflicting results (Okugawa et al., 2014; Zheng et al., 2014; Ma et al., 2015; Zhang et al., 2015). Therefore, in this meta-analysis we endeavored to evaluate the effect of MALAT1 expression on clinical pathological features and prognosis in various cancers.

\section{MATERIAL AND METHODS}

\section{Literature search}

Relevant studies published as of April 2015 were retrieved using Embase, PubMed, EBSCO, Ovid, and Web of Science databases, as well as manual searching. No restriction was placed on geographic origin, but publication language was limited to Chinese or English. The search strategy involved a combination of keywords related to MALAT1 and cancer. The following search terms were used in combinations of keywords and free words: ("long non-coding RNA, human" OR "MALAT1" OR "metastasis associated lung adenocarcinoma transcript 1" OR "MALAT-1" OR "NEAT2") and ("cancer" OR "tumor" OR "tumour*" OR "carcinoma*” OR "neoplas*” OR "malignan").

\section{Inclusion and exclusion criteria}

The following inclusion criteria were taken into account when collecting published articles for the present study: 1) patient diagnoses were confirmed and no restriction was placed on cancer type; 2) data included sex, age, tumor size and stage, World Health Organization differentiation grade, metastasis status, and overall survival (OS); 3 ) the study focus should consist of the relationship between MALAT1 expression and the clinical pathological features and prognosis of cancers; and 4) the investigation should be in the form of a cohort study. Articles that did not meet the above criteria were excluded. In addition, studies were excluded if they: 1) consisted of abstracts only; 2) were case reports; 3) were based on animal models or cell lines; (3) were duplicate publications (only the most recent or complete study was included); or 4) provided insufficient information relating to our topic of interest.

\section{Data extraction and quality assessment}

Descriptive information related to the key focus of this article was collected using a standard form containing the following fields: first author name, year of publication, country/ethnicity, cancer type(s), sample size, age, sex, tumor size, differentiation grade, tumor stage, metastasis status, and OS. The above data were extracted from each study by two independent researchers, to reduce the probability of selection bias and strengthen the reliability of this meta-analysis. Disagreement regarding the inclusion of data was settled by consultation with a third investigator.

We assessed risk of bias by examining the random sequence generation, allocation concealment, blinding (of participants, personnel, and outcome assessment), and selective outcome reporting used in each trial (Higgins and Green, 2011). Two authors (X.S. Shi and J. $\mathrm{Li}$ ) independently assessed the included studies and rated each as having low, high, or unclear risk of bias. 


\section{Statistical analysis}

Statistical calculation was performed with Stata statistical software version 12.0 (StataCorp, College Station, TX, USA). Odds ratios (ORs) and hazards ratios (HRs) with 95\% confidence intervals $(95 \% \mathrm{Cls})$ were estimated using fixed- or random-effects models to evaluate the association between MALAT1 expression and the clinical pathological features and prognosis of cancers. AZ-test was employed to assess the significance of the pooled effect size (Chen et al., 2012) and forest plots were generated to display between-group comparisons of HRs and ORs with $95 \% \mathrm{Cls}$. The $Q$ (Jackson et al., 2012) and $l^{2}$ tests were applied to assess between-study heterogeneity (Peters et al., 2006). When values of $P$ value $<0.05$ or $I^{2}>50 \%$ revealed significant heterogeneity, the random-effects model was implemented. The fixed-effects model was employed in all other cases (Zintzaras and loannidis, 2005). Sensitivity analysis was used to determine whether the removal of a single study influenced the overall outcome. Funnel plots and Egger's linear regression test were carried out to assess publication bias, thereby ensuring the reliability of our results (Egger et al., 1997; Sterne and Egger, 2001). Two-tailed tests were conducted, with $P<0.05$ signifying statistical significance.

\section{RESULTS}

\section{Selection of eligible studies}

The initial search yielded 227 articles and after screening titles and abstracts, 168 citations were excluded. Fifty-nine potentially relevant articles were selected for full-text review (Figure1). Finally, 12 primary studies, including 958 patients, met the inclusion criteria (Ji et al., 2003; Lai et al., 2012; Cho et al., 2014; Liu et al., 2014; Okugawa et al., 2014; Zheng et al., 2014; Dong et al., 2015; Hirata et al., 2015; Li et al., 2015; Ma et al., 2015; Pang et al., 2015; Zhang et al., 2015). Details regarding the participants of these studies are summarized in Table 1. Figure 2 provides an overall picture of the methodological quality of the selected investigations, as evaluated by the quality assessment tool for diagnostic accuracy studies (QUADAS; Whiting et al., 2011).

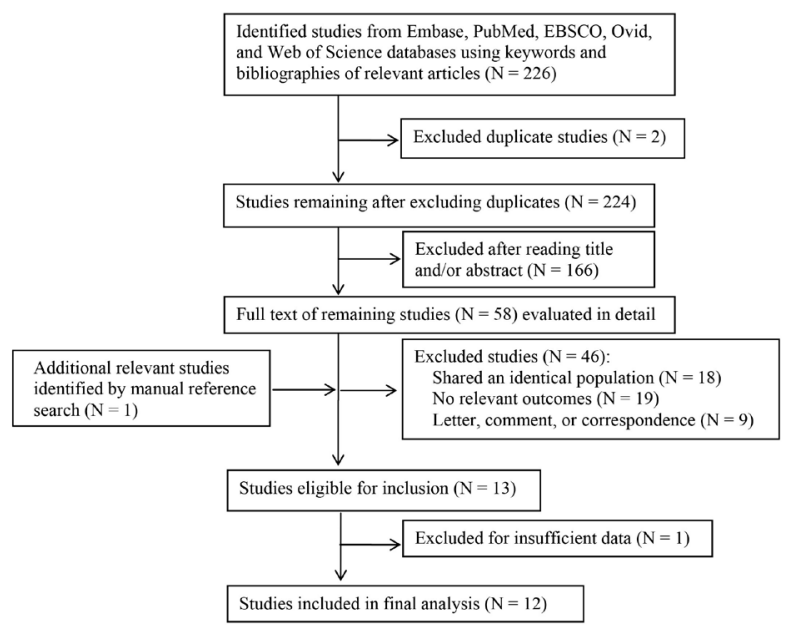

Figure 1. Flow chart of study identification and inclusion. 
X.S. Shi et al.

18812

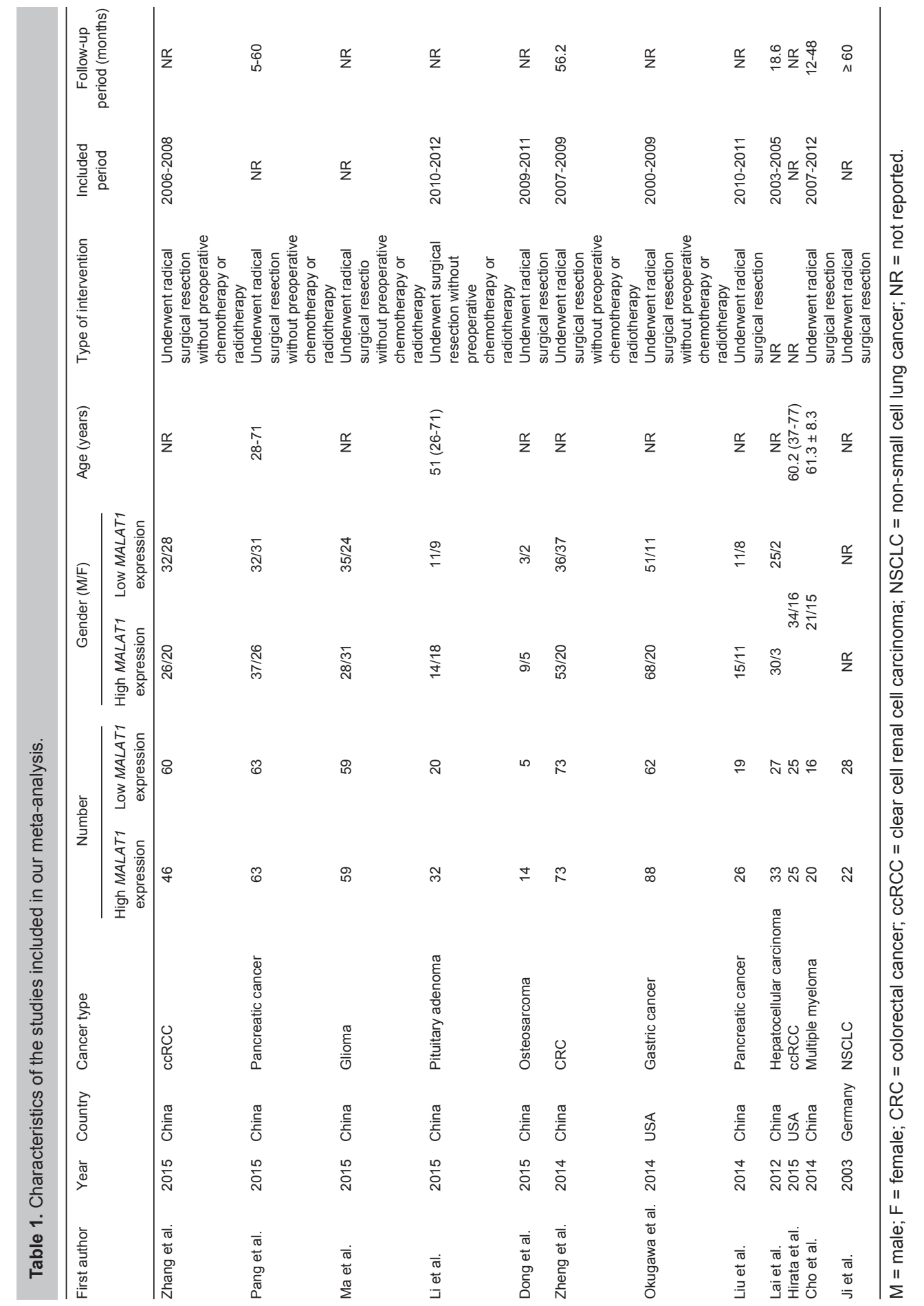




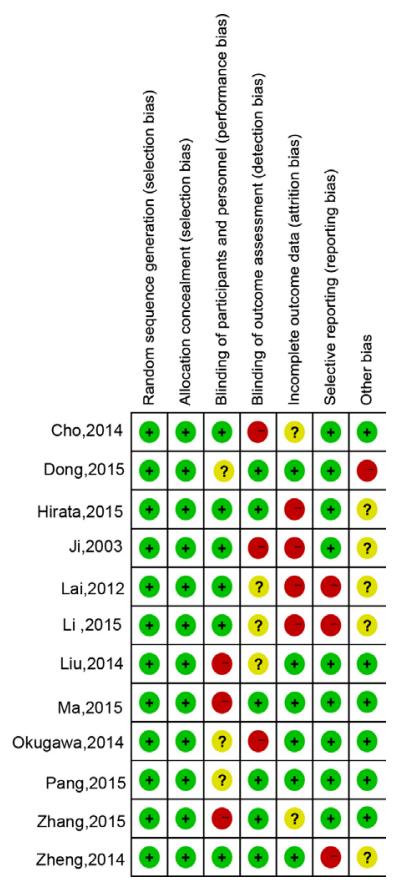

Figure 2. An overall picture of the methodological quality of the included studies, as evaluated by the quality assessment tool for diagnostic accuracy studies.

\section{Meta-analysis of the association between MALAT1 expression and human cancers}

Heterogeneity was observed in some of the data used in our meta-analysis, thus both random- and fixed-effects models were employed. We found that expression of MALAT1 was connected with tumor prognosis $(\mathrm{HR}=3.11,95 \% \mathrm{Cl}=1.98-4.23, \mathrm{P}=0.000)$, implying that it may be useful in predicting cancer outcome (Figure $3 \mathrm{~A}$ ). With respect to clinical pathological features, patients overexpressing MALAT1 had larger tumors than those showing low expression (OR $=0.40$, $95 \% \mathrm{Cl}=0.21-0.74, \mathrm{P}=0.003$; Figure $3 \mathrm{~B}$ ), but no connection was found between MALAT1 level and tumor differentiation, T-stage, lymph node (LN) metastasis, or distant metastasis (all P > 0.05; Figures $3 \mathrm{C}$ to $3 \mathrm{~F})$.

\section{Subgroup analysis of the association between MALAT1 expression and human cancers}

Subgroup analysis was performed based on sample size. We observed a clear correlation between increased MALAT1 expression and poor cancer prognosis in both the large $(\mathrm{HR}=2.75$, $95 \% \mathrm{Cl}=1.92-3.57, \mathrm{P}=0.000$; Figure $4 \mathrm{~A})$, and small sample-size subgroup $(\mathrm{HR}=4.22,95 \% \mathrm{Cl}=$ $0.04-8.40, P=0.048)$. Concerning clinical features, increased expression was found to be related to tumor size in patients from studies with larger sample sizes $(\mathrm{OR}=0.30,95 \% \mathrm{Cl}=0.13-0.71, \mathrm{P}=$ 0.006; Figure 4B), but no such connection was discerned using the small sample-size dataset $(\mathrm{P}=$ 0.246). In addition, elevated MALAT1 expression was associated with T-stage in the small samplesize subset $(\mathrm{OR}=0.14,95 \% \mathrm{Cl}=0.04-0.55, \mathrm{P}=0.005$; Figure 4D). Elevated MALAT1 expression 
was also associated with distant metastasis in the small sample-size subset $(\mathrm{OR}=0.13,95 \% \mathrm{Cl}=$ 0.03-0.70, $\mathrm{P}=0.017$; Figure 4F). However, no link between MALAT1 levels and tumor differentiation, LN metastasis was apparent in either subgroup (all $\mathrm{P}>0.05$; Figures 4C, 4E).

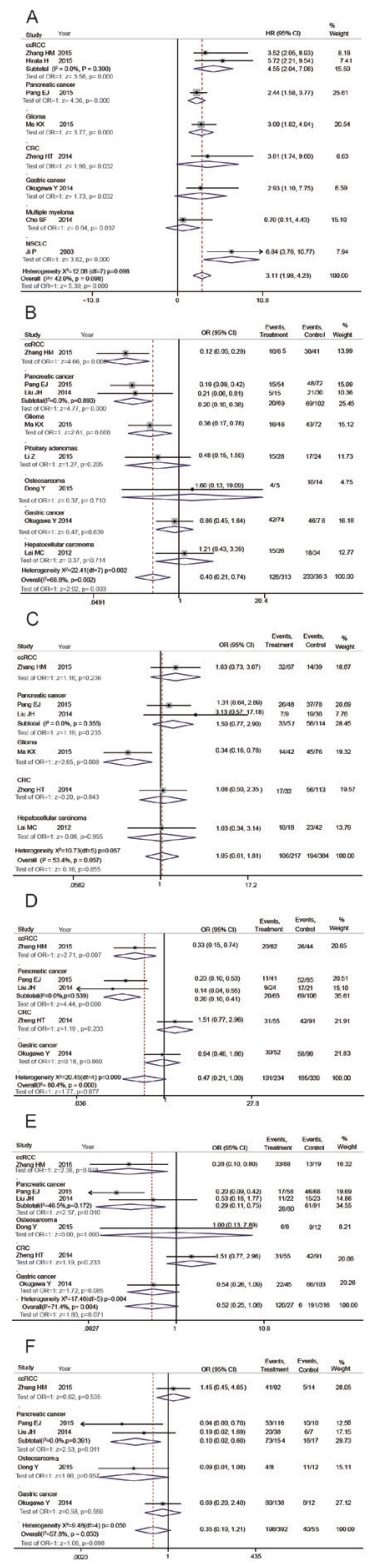

Figure 3. Forest plots of the relationship between MALAT1 expression and clinical pathological features and prognosis of cancers. Individual and pooled hazard ratios (HRs) and odds ratios (ORs) with $95 \%$ confidence intervals (Cls) regarding the association between MALAT1 expression and (A) prognosis, (B) tumor size, (C) histological grade, (D) T-stage, $(E)$ lymph node metastasis, and $(F)$ distant metastasis are shown. ccRCC = clear cell renal cell carcinoma; $\mathrm{CRC}=$ colorectal cancer; $\mathrm{NSCLC}=$ non-small cell lung cancer; $\mathrm{df}=$ degrees of freedom. 


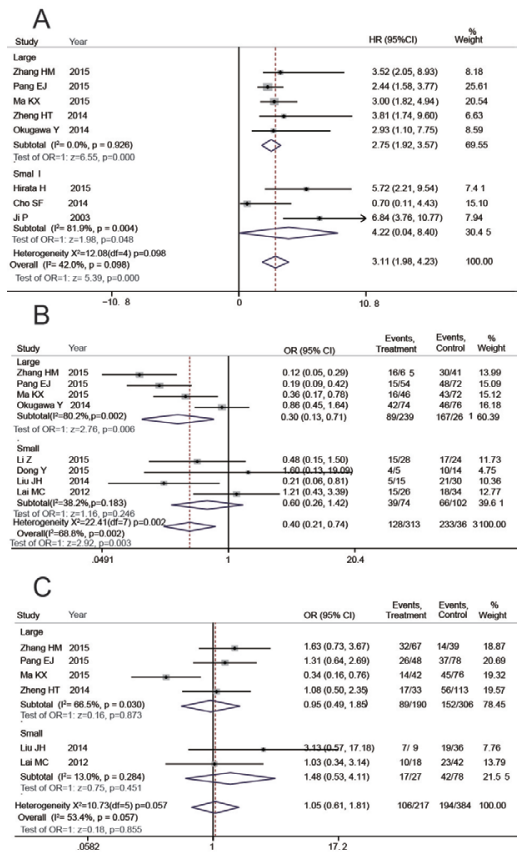

D
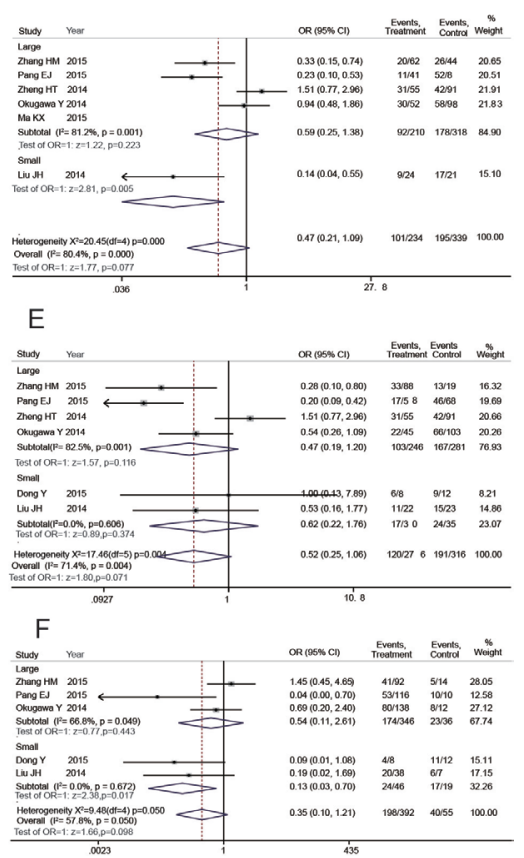

Figure 4. Sample-size subgroup analysis of the relationship between MALAT1 expression and clinical pathological features and prognosis of cancers. Individual and pooled hazard ratios (HRs) and odds ratios (ORs) with $95 \%$ confidence intervals (Cls) regarding the association between MALAT1 expression and (A) prognosis, $(\mathrm{B})$ tumor size, $(\mathrm{C})$ histological grade, (D) T-stage, (E) lymph node metastasis, and (F) distant metastasis are shown. $\mathrm{df}=$ degrees of freedom. 


\section{Publication bias}

No obvious asymmetry was distinguished in the funnel plots, and in accordance with these results, Egger's regression test revealed no publication bias in all six analyses (all P > 0.05; Figure 5).
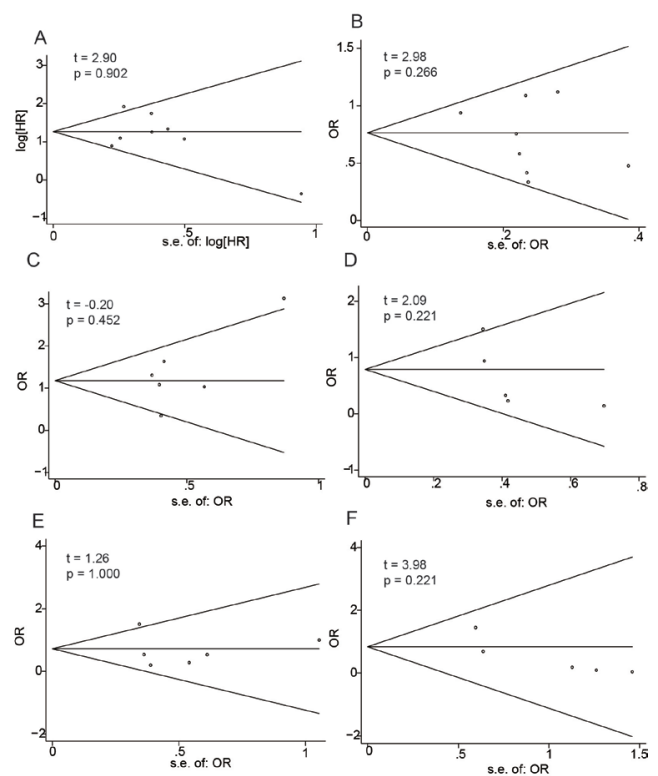

Figure 5. Funnel plots assessing publication bias in our meta-analysis of the association between MALAT1 expression and (A) cancer prognosis, (B) tumor size, (C) histological grade, (D) T-stage, (E) lymph node metastasis, and (F) distant metastasis. $\mathrm{HR}=$ hazard ratio; $\mathrm{OR}=$ odds ratio; $\mathrm{SE}=$ standard error.

\section{DISCUSSION}

In the current meta-analysis, we systematically investigated the link between MALAT1 expression and the clinical pathological characteristics and prognosis of various malignancies. Our results suggest that the expression of MALAT1 is correlated with tumor prognosis, implying that it might constitute an important indicator of cancer out come. However, the mechanism behind this association has not yet been elucidated. Therefore, we suggest here some of the processes that may be responsible. Previous studies have indicated that MALAT1 may enhance cell proliferation and plays a key role in tumorigenesis, and when its expression is knocked down in cancer cells, ontogenesis is significantly impaired (Tano et al., 2010; Zheng et al., 2014). Furthermore, elevated expression of this IncRNA appears to accelerate the migration and epithelial to mesenchymal transition of tumor cells by activating the Wnt pathway (Ying et al., 2012). Considering this, it is possible to conclude that MALAT1 expression exerts an important influence on cancer outcome. Consistent with our results, Pang et al. (2015) also suggested that overexpression of MALAT1 might be a reliable biomarker of unfavorable prognosis in cancer patients.

The results of our investigation into the relationship between MALAT1 and clinical pathological features revealed that patients over expressing this gene had larger tumors than those demonstrating low expression, but no connection was established with histological grade, T-stage, LN metastasis, or distant metastasis. This may be partially explained by the fact that MALAT1 
regulates the levels of active serine/argentine splicing factors, and thus modulates the splicing of pre-mRNAs, thereby performing significant part in cancer formation and invasion (Dong et al., 2015). It has been shown that MALAT1 participates in the regulation of cell cycle progression and the genesis and growth of tumors by interfering with E2F1 activity (Tripathi et al., 2013; Zheng et al., 2014). Another possible explanation was provided by a previous study in which knockdown of MALAT1 appeared to strongly reduce PI3Kp85a and phosphorylatedAkt expression (Dong et al., 2015). It is widely accepted that the PI3K/Akt pathway is a vital signal transduction mechanism, and by activating the PI3K/Akt signaling cascade, MALAT1 may promote rapid tumor growth and invasion (Liu et al., 2008; Dong et al., 2015).

In order to further understand the role of MALAT1 expression in cancer, subgroup analysis was conducted based on sample size. We observed a close correlation between MALAT1 expression and poor prognosis in the large and small sample-size subgroups. With regard to clinical features, we found that the level of this IncRNA was related to tumor size in patients from studies with larger sample sizes, and with patient T-stage and distant metastasis in small sample-size investigations. In addition, no significant association with histological grade, LN metastasis was established in either subgroup. In conclusion, the overall results of our meta-analysis were in accordance with the principal findings of previous studies, suggesting that measurement of MALAT1 expression may be useful in evaluating the prognosis and clinical pathological characteristics of cancer patients.

Some limitations need to be taken into account when interpreting our results. First, the cutoff values used to define categories of MALAT1 expression differed between studies, potentially introducing bias. All factors that may influence the pooled results should therefore be carefully considered. Second, although no publication bias was evident in this analysis; most studies tend to report positive, rather than negative, results. In addition, as we restricted the publication languages to English and Chinese, relevant articles that may have met our inclusion criteria could have been overlooked, representing a potential source of selection bias. Third, the majorities of studies included (12) were performed in Asia, which may have led to selection bias, and influences the broader applicability of our results. Finally, although various clinical pathological measurements were included, only tumor size was confirmed to be positively correlated with MALAT1 expression. Moreover, the statistical analysis for several clinical parameters incorporated only one or two studies, possibly affecting the reliability of the final results. The heterogeneity of study designs may be responsible for this issue.

Taken together, our findings clarify the significance of MALAT1 as an important clinical biomarker of poor prognosis and adverse pathological features in cancer patients. These results could have significant value in pathological examination and outcome prediction, as well as providing a new insight for the selection of therapeutic approaches in clinical application. However, the present results should be interpreted cautiously due to the above limitations, and the implementation of further large sample-size, multicenter studies would assist in clarifying this matter.

\section{Conflicts of interest}

The authors declare no conflict of interest.

\section{ACKNOWLEDGMENTS}

Research supported in part by the National Natural Science Foundation of China (\#81201845). We would like to acknowledge the reviewers for their helpful comments concerning this paper. 


\section{REFERENCES}

Astin M, Griffin T, Neal RD, Rose P, et al. (2011). The diagnostic value of symptoms for colorectal cancer in primary care: a systematic review. Br. J. Gen. Pract. 61: e231-243.

Axon A (2006). Symptoms and diagnosis of gastric cancer at early curable stage. Best Pract. Res. Clin. Gastroenterol. 20: 697-708.

Chen H, Manning AK and Dupuis J (2012). A method of moments estimator for random effect multivariate meta-analysis. Biometrics 68: 1278-1284.

Cho SF, Chang YC, Chang CS, Lin SF, et al. (2014). MALAT1 long non-coding RNA is overexpressed in multiple myeloma and may serve as a marker to predict disease progression. BMC Cancer 14: 809.

Dong Y, Liang G, Yuan B, Yang C, et al. (2015). MALAT1 promotes the proliferation and metastasis of osteosarcoma cells by activating the PI3K/Akt pathway. Tumour Biol. 36: 1477-1486.

Egger M, Davey Smith G, Schneider M and Minder C (1997). Bias in meta-analysis detected by a simple, graphical test. BMJ 315: 629-634.

Gao W, Shen H, Liu L, Xu J, et al. (2011). MiR-21 overexpression in human primary squamous cell lung carcinoma is associated with poor patient prognosis. J. Cancer Res. Clin. Oncol. 137: 557-566.

Gutschner T, Hammerle M, Eissmann M, Hsu J, et al. (2013). The noncoding RNA MALAT1 is a critical regulator of the metastasis phenotype of lung cancer cells. Cancer Res. 73: 1180-1189.

Higgins JPT and Green S (editors) 2011. Cochrane handbook for systematic reviews of interventions. Version 5.1.0. The Cochrane Collaboration. Available from www.cochrane-handbook.org.

Hirata H, Hinoda Y, Shahryari V, Deng G, et al. (2015). Long noncoding RNA MALAT1 promotes aggressive renal cell carcinoma through Ezh2 and interacts with miR-205. Cancer Res. 75: 1322-1331.

Jackson D, White IR and Riley RD (2012). Quantifying the impact of between-study heterogeneity in multivariate metaanalyses. Stat. Med. 31: 3805-3820.

Ji P, Diederichs S, Wang W, Boing S, et al. (2003). MALAT-1, a novel noncoding RNA, and thymosin beta4 predict metastasis and survival in early-stage non-small cell lung cancer. Oncogene 22: 8031-8041.

Lai MC, Yang Z, Zhou L, Zhu QQ, et al. (2012). Long non-coding RNA MALAT-1 overexpression predicts tumor recurrence of hepatocellular carcinoma after liver transplantation. Med. Oncol. 29: 1810-1816.

Lapunzina P, Lopez RO, Rodriguez-Laguna L, Garcia-Miguel P, et al. (2014). Impact of NGS in the medical sciences: Genetic syndromes with an increased risk of developing cancer as an example of the use of new technologies. Genet. Mol. Biol. 37: 241-249.

Leong DT and Ng KW (2014). Probing the relevance of 3D cancer models in nanomedicine research. Adv. Drug Deliv. Rev. 79-80: 95-106.

Li Z, Li C, Liu C, Yu S, et al. (2015). Expression of the long non-coding RNAs MEG3, HOTAIR, and MALAT-1 in non-functioning pituitary adenomas and their relationship to tumor behavior. Pituitary 18: 42-47.

Liu B, Shi ZL, Feng J and Tao HM (2008). Celecoxib, a cyclooxygenase-2 inhibitor, induces apoptosis in human osteosarcoma cell line MG-63 via down-regulation of PI3K/Akt. Cell Biol. Int. 32: 494-501.

Liu JH, Chen G, Dang YW, Li CJ, et al. (2014). Expression and prognostic significance of IncRNA MALAT1 in pancreatic cancer tissues. Asian Pac. J. Cancer Prev. 15: 2971-2977.

Ma KX, Wang HJ, Li XR, Li T, et al. (2015). Long noncoding RNA MALAT1 associates with the malignant status and poor prognosis in glioma. Tumour Biol. 36: 3355-3359.

Okugawa Y, Toiyama Y, Hur K, Toden S, et al. (2014). Metastasis-associated long non-coding RNA drives gastric cancer development and promotes peritoneal metastasis. Carcinogenesis 35: 2731-2739.

Pang EJ, Yang R, Fu XB and Liu YF (2015). Overexpression of long non-coding RNA MALAT1 is correlated with clinical progression and unfavorable prognosis in pancreatic cancer. Tumour Biol. 36: 2403-2407.

Peters JL, Sutton AJ, Jones DR, Abrams KR, et al. (2006). Comparison of two methods to detect publication bias in metaanalysis. JAMA 295: 676-680.

Sterne JA and Egger M (2001). Funnel plots for detecting bias in meta-analysis: guidelines on choice of axis. J. Clin. Epidemiol. 54: 1046-1055.

Tano K, Mizuno R, Okada T, Rakwal R, et al. (2010). MALAT-1 enhances cell motility of lung adenocarcinoma cells by influencing the expression of motility-related genes. FEBS Lett. 584: 4575-4580.

Tripathi V, Ellis JD, Shen Z, Song DY, et al. (2010). The nuclear-retained noncoding RNA MALAT1 regulates alternative splicing by modulating SR splicing factor phosphorylation. Mol. Cell 39: 925-938.

Tripathi V, Shen Z, Chakraborty A, Giri S, et al. (2013). Long noncoding RNA MALAT1 controls cell cycle progression by regulating the expression of oncogenic transcription factor B-MYB. PLoS Genet. 9: e1003368. 
Whiting PF, Rutjes AW, Westwood ME, Mallett S, et al. (2011). QUADAS-2: a revised tool for the quality assessment of diagnostic accuracy studies. Ann. Intern. Med. 155: 529-536.

Yamashita T and Wang XW (2013). Cancer stem cells in the development of liver cancer. J. Clin. Invest. 123: 1911-1918.

Yan LX, Huang XF, Shao Q, Huang MY, et al. (2008). MicroRNA miR-21 overexpression in human breast cancer is associated with advanced clinical stage, lymph node metastasis and patient poor prognosis. RNA 14: 2348-2360.

Ying L, Chen Q, Wang Y, Zhou Z, et al. (2012). Upregulated MALAT-1 contributes to bladder cancer cell migration by inducing epithelial-to-mesenchymal transition. Mol. Biosyst. 8: 2289-2294.

Zhang HM, Yang FQ, Chen SJ, Che J, et al. (2015). Upregulation of long non-coding RNA MALAT1 correlates with tumor progression and poor prognosis in clear cell renal cell carcinoma. Tumour Biol. 36: 2947-2955.

Zheng HT, Shi DB, Wang YW, Li XX, et al. (2014). High expression of IncRNA MALAT1 suggests a biomarker of poor prognosis in colorectal cancer. Int. J. Clin. Exp. Pathol. 7: 3174-3181.

Zintzaras E and loannidis JP (2005). Heterogeneity testing in meta-analysis of genome searches. Genet. Epidemiol. 28: 123-137. 